\title{
Dynamics of a Family of Piecewise-Linear Area-Preserving Plane Maps III. Cantor Set Spectra
}

\author{
Jeffrey C. Lagarias \\ Department of Mathematics \\ University of Michigan \\ Ann Arbor, MI 48109-1043 \\ email: lagarias@umich.edu \\ Eric Rains \\ Dept. of Mathematics \\ University of California, Davis \\ Davis, CA 95616-8633 \\ email: rains@math.davis.edu
}

(August 3, 2006 version)

\begin{abstract}
This paper studies the behavior under iteration of the maps $T_{a b}(x, y)=\left(F_{a b}(x)-y, x\right)$ of the plane $\mathbb{R}^{2}$, in which $F_{a b}(x)=a x$ if $x \geq 0$ and $b x$ if $x<0$. These maps are area-preserving homeomorphisms of $\mathbb{R}^{2}$ that map rays from the origin into rays from the origin. Orbits of the map correspond to solutions of the nonlinear difference equation $x_{n+2}=1 / 2(a-b)\left|x_{n+1}\right|+$ $1 / 2(a+b) x_{n+1}-x_{n}$. This difference equation can be rewritten in an eigenvalue form for a nonlinear difference operator of Schrödinger type $-x_{n+2}+2 x_{n+1}-x_{n}+V_{\mu}\left(x_{n+1}\right) x_{n+1}=E x_{n+1}$, in which $\mu=\frac{1}{2}(a-b)$ is fixed, and $V_{\mu}(x)=\mu(\operatorname{sgn}(x))$ is an antisymmetric step function potential, and the energy $E=2-\frac{1}{2}(a+b)$. We study the set $\Omega_{S B}$ of parameter values where the map $T_{a b}$ has at least one bounded orbit, which correspond to $l_{\infty}$-eigenfunctions of this difference operator. The paper shows that for transcendental $\mu$ the set $\operatorname{Spec}_{\infty}[\mu]$ of energy values $E$ having a bounded solution is a Cantor set. Numerical simulations suggest the possibility that these Cantor sets have positive (one-dimensional) measure for all real values of $\mu$.
\end{abstract}

Keywords: area preserving map, discrete Schrödinger operator, symbolic dynamics, tight binding model

AMS Subject Classification: Primary: 37E30 Secondary: 52C23, 82D30 


\section{Introduction}

As in parts I and II, we study the behavior under iteration of the two parameter family of piecewise-linear homeomorphisms of $\mathbb{R}^{2}$ given by

$$
T_{a b}(x, y)= \begin{cases}(a x-y, x) & \text { if } \quad x \geq 0 \\ (b x-y, x) & \text { if } x<0\end{cases}
$$

The parameter space is $(a, b) \in \mathbb{R}^{2}$. This map can be written

$$
T_{a b}(x, y)=\left[\begin{array}{cc}
F_{a b}(x) & -1 \\
1 & 0
\end{array}\right]\left[\begin{array}{l}
x \\
y
\end{array}\right]
$$

in which

$$
F_{a b}(x)=\left\{\begin{array}{lll}
a & \text { if } & x \geq 0 \\
b & \text { if } & x<0
\end{array}\right.
$$

The formula (1.2) shows that $T_{a b}(x, y)$ is a homeomorphism, since

$$
T_{a b}^{-1}(x, y)=\left[\begin{array}{cc}
F_{a b}(y) & -1 \\
1 & 0
\end{array}\right]^{-1}\left[\begin{array}{l}
x \\
y
\end{array}\right]=\left[\begin{array}{cc}
0 & 1 \\
-1 & F_{a b}(y)
\end{array}\right]\left[\begin{array}{l}
x \\
y
\end{array}\right]
$$

and it preserves the area form $d \omega=d x \wedge d y$. In part I we observed that iterating this map encodes the solutions of the second-order nonlinear recurrence

$$
x_{n+2}=\mu\left|x_{n+1}\right|+\nu x_{n+1}-x_{n}
$$

via

$$
T_{a b}\left(x_{n+1}, x_{n}\right)=\left(x_{n+2}, x_{n+1}\right)
$$

in which

$$
\mu=\frac{1}{2}(a-b), \quad \nu:=\frac{1}{2}(a+b)
$$

or equivalently

$$
a=\nu+\mu, \quad b=\nu-\mu .
$$

This recurrence can be rewritten as a one-dimensional discrete nonlinear difference equation of Schrödinger type

$$
-x_{n+2}+2 x_{n+1}-x_{n}+V_{\mu}\left(x_{n+1}\right) x_{n+1}=E x_{n+1},
$$

where the potential $V_{\mu}(x)$ is given by

$$
V_{\mu}(x):=\left\{\begin{array}{cl}
\mu & \text { if } \quad x \geq 0 \\
-\mu & \text { if } \quad x<0
\end{array}\right.
$$

and the energy value $E$ is given by

$$
E:=2-\nu
$$

Holding the potential $V_{\mu}$ fixed and letting the parameter $\nu$ vary amounts to studying the set of solutions for all energy values $E=2-\nu$. 
The values of $E$ corresponding to bounded orbits are analogous to the $l_{\infty}$-spectrum of discrete linear Schrödinger operators

$$
\Phi_{V}(x)_{n+1}=-x_{n+2}+2 x_{n+1}-x_{n}+V(n+1) x_{n+1} .
$$

in which linearity is reflected in the potential $V(n)$ depending only on $n$ and not on $x_{n}$. The model (1.11) is often called the "tight-binding" approximation to the Schrödinger operator on the line. For a bounded potential $\Phi_{V}$ it gives a well-defined bounded operator on all sequence spaces $l_{p}(\mathbb{Z})$. The eigenvalue equation is

$$
\Phi(x)_{n+1}=-x_{n+2}+2 x_{n+1}-x_{n}+V(n+1) x_{n+1}=E x_{n+1} .
$$

In this context one is interested in characterizing the values of $E$ which have an orbit of the following types.

(1) Extended state. The orbit $\left\{x_{n}:-\infty \leq n \leq \infty\right\}$ is a bounded orbit, i.e. lies in $l_{\infty}(\mathbb{Z})$.

(2) Localized state. The orbit $\left\{x_{n}:-\infty \leq n \leq \infty\right\}$ lies in $l_{2}(\mathbb{Z})$.

The energy values $E$ for which there exists an orbit of type (1) comprise the $l_{\infty}$-spectrum

$$
\operatorname{Spec}_{\infty}[\mu]:=\left\{E=2-\nu:(\mu, \nu) \in \Omega_{b d d}\right\} .
$$

A weaker version of (2) is the topological property that the orbit $\left\{x_{n}:-\infty \leq n \leq \infty\right\}$ satisfies

$$
\lim _{n \rightarrow \pm \infty} x_{n}=0
$$

we call such an orbit weakly localized.

Much work on the discrete linear Schrödinger operator was motivated by the observation of Hofstadter [9] in 1976 that for a quasiperiodic linear potential $V(n)=\lambda \cos (2 \pi \alpha n)$ with $\lambda=2$ there is an $l_{\infty}$ eigenvalue structure $\Sigma_{\lambda, \alpha}$ which when (numerically) plotted for variable $\alpha$ appeared to form a fractal "butterfly". More precisely, for fixed irrational $\alpha$ the $l_{\infty}$-spectrum appeared to be a Cantor set of measure zero. Hofstadter's model has been much studied, and the Hofstadter "butterfly" has been justified to some extent. This has been done particularly in the context of the $l^{2}$-spectrun of the almost Mathieu equation, see Jitomirskaya [10] and Puig [16] for recent results. Further references are given at the end of the introduction.

Here we study analogous questions for the for the nonlinear difference operator of Schrödinger type (1.8). We obtain rigorous results about the $l_{\infty}$ spectrum by exploiting the piecewise-linear structure of the maps. The object of this paper is to determine structural properties of the set

$$
\Omega_{S B}:=\left\{(\mu, \nu): T_{\mu \nu} \text { has at least one nonzero bounded orbit }\right\},
$$

where $S B=$ "semi-bounded". We also obtain results concerning the set of parameter values with all orbits bounded, which we denote

$$
\Omega_{B}:=\left\{(\mu, \nu): T_{\mu \nu} \text { has all nonzero orbits bounded }\right\} .
$$

Clearly $\Omega_{B} \subseteq \Omega_{S B}$, and Theorem 2.1(1) of this paper implies that the inclusion is strict. The set $\Omega_{S B}$ is an analogue of the Hofstadter "butterfly" set in our context. We prove that $\Omega_{S B}$ is a closed set, and present evidence for the following conjecture. 
Conjecture B. The set $\Omega_{S B}$ has positive two-dimensional Lebesgue measure. Furthermore, for each real $\mu$ the $l_{\infty}$-spectrum

$$
\operatorname{Spec}_{\infty}[\mu]:=\left\{E=2-\nu:(\mu, \nu) \in \Omega_{S B}\right\}
$$

has positive one-dimensional Lebesgue measure.

The main result of the paper is to prove that the set $\operatorname{Spec}_{\infty}[\mu]$ is a Cantor set (totally disconnected perfect set) for all parameter values $\mu$ outside an exceptional set $\mathcal{E}$ consisting entirely of algebraic numbers (Theorem 2.4). Thus these sets $\operatorname{Spec}_{\infty}[\mu]$ exhibit a property ascribed to the Hofstadter "butterfly". The value $\mu=0$ is exceptional, and $S p e c_{\infty}[0]$ is the entire interval $[0,4]$. As far as we know $\mu=0$ might be the only value in the exceptional set; if so the set $\Omega_{S B}$ for $\mu>0$ would have the structure (Cantor set) $\times$ (line). We present numerical evidence suggesting that for all values of $\mu$ (including the exceptional values) the set $S p e c_{\infty}[\mu]$ has positive one-dimensional Lebesgue measure. If true, this would contrast with the Hofstadter "butterfly".

The main difficulty in the proof of Theorem 2.4 is to show that for those $\mu$ that are not algebraic numbers the set $\operatorname{Spec}_{\infty}[\mu]$ is totally disconnected. This reduces to showing that there are a dense set of rationals in $[0,1 / 2]$ that have nondegenerate rotation intervals. Establishing this requires nontrivial argument, for part I showed that for all non-algebraic $\mu$ infinitely many rational rotation numbers always have degenerate rotation intervals, namely $r=\frac{2 n-1}{4 n}$ for $n \geq 2$. The main part of the proof of Theorem 2.4 shows that for each transcendental number $\mu$ all rational rotation numbers $\frac{k}{p}$ in $\left(0, \frac{1}{2}\right)$ with a prime denominator $p$ have nondegenerate rotation intervals (Theorem 4.2). The arguments used have a number-theoretic flavor.

In $\S 2$ we state the main results, and establish them in $\S 3-\S 4$. In $\S 5$ we make some concluding remarks and formulate open questions.

Prior work on these maps includes Herman [8, VIII], and Beardon, Bullett and Rippon [2]. Both these works studied, among other things, the set $\Omega_{B}$ where all orbits are bounded. Their results were discussed in parts I and II, and we remark on them later in this paper.

The results of this paper may be compared with various results concerning linear difference Schrödinger operators. For general references on spectra of linear difference Schrödinger operators see Bougerol and Lacroix [5] and Pasteur and Pigotin [15]. For discussion of existence or nonexistence of Cantor set spectra for various potentials see Bellissard [3], Fröhlich et al [6] and the earlier references for the almost Mathieu equation. For Schrödinger operator potentials taking finitely many values, see Kotani [1], Sutherland and Kohmoto [19] and Sütö[20].

Notation. We write $\mathbf{v}=\left(\mathbf{v}_{x}, \mathbf{v}_{y}\right) \in \mathbb{R}^{2}$, to be viewed as a column vector. An interval $\left[\mathbf{v}_{1}, \mathbf{v}_{2}\right)$ of the unit circle, or a corresponding sector $\mathbb{R}^{+}\left[\mathbf{v}_{1}, \mathbf{v}_{2}\right)$ of the plane $\mathbb{R}^{2}$, is specified by rotating counterclockwise from $\mathbf{v}_{1}$ to $\mathbf{v}_{2}$. We let $\operatorname{Meas}_{d}(S)$ denote the $d$-dimensional Lebesgue measure of a set $S$, for $d=1,2$.

Acknowledgment. We did much of the work on this paper while employed at AT\&T LabsResearch, whom we thank for support. We thank Jim Reeds for suggesting a proof method for Lemma 4.1. We thank M. Kontsevich for bringing the work of Bedford, Bullett and Rippon [2] to our attention. 


\section{Summary of Results}

The parameter space of the map can be taken to be either $(a, b)$ or $(\mu, \nu)$, as these are equivalent by

$$
\mu=\frac{1}{2}(a-b), \quad \nu=\frac{1}{2}(a+b) .
$$

Both coordinate systems have their advantages, and we write the map (1.1) as $T_{a b}, T_{\mu \nu}$ accordingly. We will also use the mixed parameter space $(a, \mu)$. It is convenient to represent the action of $T_{a b}$, acting on row vectors $\mathbf{v}_{n}=\left(x_{n+1}, x_{n}\right)$ as

$$
T_{n}\left(\mathbf{v}_{0}\right)=\left(x_{n+1}, x_{n}\right)=M_{n}\left(\mathbf{v}_{0}\right)\left(x_{1}, x_{0}\right)
$$

in which

$$
M_{n}\left(\mathbf{v}_{0}\right)=\prod_{i=1}^{n}\left[\begin{array}{cc}
F_{a b}\left(x_{i}\right) & -1 \\
1 & 0
\end{array}\right]:=\left[\begin{array}{cc}
F_{a b}\left(x_{n}\right) & -1 \\
1 & 0
\end{array}\right] \cdots\left[\begin{array}{cc}
F_{a b}\left(x_{2}\right) & -1 \\
1 & 0
\end{array}\right]\left[\begin{array}{cc}
F_{a b}\left(x_{1}\right) & -1 \\
1 & 0
\end{array}\right] .
$$

Conjugation by the involution $J_{0}:(x, y) \rightarrow(-x,-y)$ gives

$$
T_{b a}=J_{0}^{-1} \circ T_{a b} \circ J_{0} \text {. }
$$

Thus, in studying dynamics, without loss of generality we can restrict to the closed half-space $\{(a, b): a \geq b\}$ of the $(a, b)$ parameter space. This corresponds to the region $\{(\mu, \nu): \mu \geq 0\}$ of the $(\mu . \nu)$ parameter space, with $T_{\mu \nu}$ conjugate to $T_{-\mu, \nu}$.

The associated rotation map $S_{\mu \nu}: S^{1} \rightarrow S^{1}$ is given by

$$
S_{\mu \nu}\left(e^{i \theta}\right):=\frac{T_{\mu \nu}\left(e^{i \theta}\right)}{\left|T_{\mu \nu}\left(e^{i \theta}\right)\right|}
$$

It has a well-defined rotation number $r\left(S_{\mu \nu}\right)$, which is a counterclockwise rotation, and was shown in part I to always lie in the closed interval $[0,1 / 2]$.

In $\S 3$ we establish properties of the sets $\Omega_{S B}$ and $\Omega_{B}$.

Theorem 2.1 The set $\Omega_{S B}$ is a closed set. It consists of all parameter values $(\mu, \nu)$ for which the associated values $(a, b)$ satisfy one of the conditions below.

(1) $r\left(S_{a b}\right)$ is rational, and $T_{a b}$ has a periodic orbit.

(2) $r\left(S_{a b}\right)$ is irrational.

Case (1) was already established in part I, Theorem 2.4(i) and (iii). To handle case (2), we study the $(\mu, \nu)$-parameterization for constant $\mu$, and show the following facts. (Theorem 3.2)

(a) The set of values $\nu$ with $r\left(S_{\mu \nu}\right)=r$ with $0<r=\frac{p}{q}<\frac{1}{2}$ is either a single point $\mathbf{v}$ or a closed interval $\left[\mathbf{v}_{1}, \mathbf{v}_{2}\right]$. If it is a point then $T_{\mu \nu}$ is periodic. If it is an interval $\left[\mathbf{v}^{-}(r), \mathbf{v}^{+}(r)\right]$ then no $T_{\mu \nu}$ is periodic, and the only $T_{\mu \nu}$ with a periodic orbit are the endpoints $\mathbf{v}=\mathbf{v}^{-}(r)$ and $\mathbf{v}^{+}(r)$.

(b) The set of values $\nu$ with $r\left(S_{\mu \nu}\right)=r$ irrational with $0<r<\frac{1}{2}$ is a single point $\mathbf{v}$.

We then obtain a bounded orbit in case (b) by a limiting procedure using bounded orbits in case (a) with suitable rational rotations $\frac{p_{n}}{q_{n}}$ approaching $r$. The endpoints of rotation intervals of positive length in (a) give points in $\Omega_{S B}$ that are not in $\Omega_{B}$.

We obtain the following information on the location of $\Omega_{S B}$ viewed in the ( $\left.\mu . a\right)$ parameter space, using results from $\S 3$ of part I. 
Theorem 2.2 The set of values for which $T_{\mu \nu}$ has a bounded orbit (i.e. $(\mu, \nu) \in \Omega_{S B}$ ), in the range $\mu \geq 0$ lies inside the cylinder $-2 \leq a \leq 2$, where $a=\nu+\mu$. Furthermore:

(1) For $-2 \leq a<0$, one has

$$
0 \leq \mu \leq \frac{2}{|a|}-\frac{|a|}{2}
$$

(2) For $0 \leq a \leq 2$, set $a=2 \cos \theta$. Then for for each $n \geq 2$ and $\frac{\pi}{n+1} \leq \theta<\frac{\pi}{n}$, there holds

$$
0 \leq \mu \leq \cos \theta-\frac{\sin n \theta+\sin \theta}{\sin (n+1) \theta}
$$

A weaker bound for $\Omega_{B}$ was previously obtained by Beardon, Bullett and Rippon [2, Theorem 1.1(iii)]. In Figure 2.1 below we plot the parameter region allowed in Theorem 2.2 in the $(\mu, a)$ plane, with $\mu=\frac{1}{2}(a-b)$, for $0 \leq \mu \leq 6$. The region $\mathcal{R}$ of Theorem 2.2 becomes unbounded

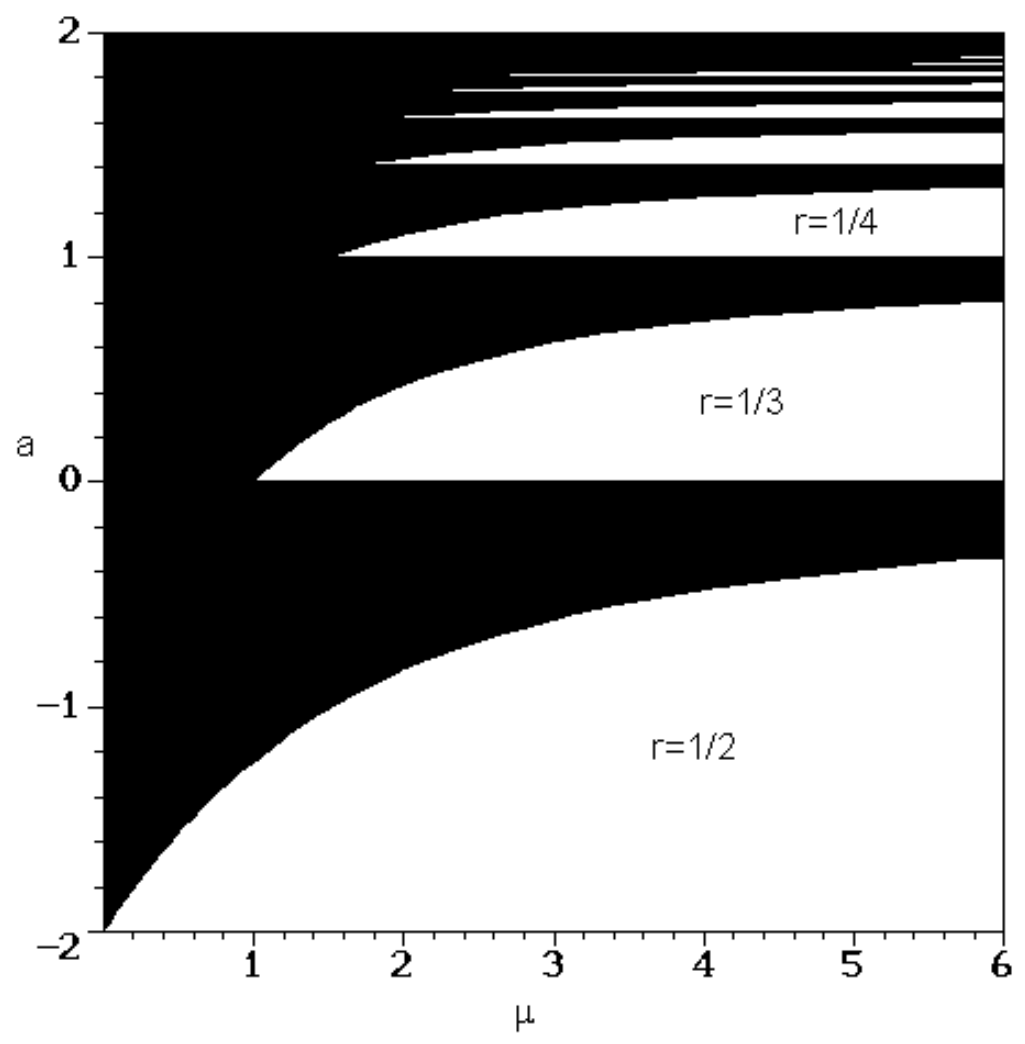

Figure 2.1: Region $\mathcal{R}$ containing $\Omega_{S B}$ in $(a, \mu)$-plane.

along the lines

$$
a=a_{n}=2 \cos \frac{\pi}{n}, \quad n \geq 2,
$$

which are $a=0,1, \sqrt{2}, 2 \cos \frac{\pi}{5}, \sqrt{3} \ldots$ 
The excluded regions in the strip $-2 \leq a \leq 2$ touching $a_{n}$ for $n \geq 1$ are regions where $T_{\mu \nu}$ has rational rotation number $r_{n}=\frac{1}{n}$. At the end of $\S 3$ we present evidence that the set $\Omega_{S B}$ has positive Lebesgue measure, and that each $\operatorname{Spec}_{\infty}[\mu]$ has positive one-dimensional Lebesgue measure. The bounds of Theorem 2.2 imply that

$$
\lim _{\mu \rightarrow \infty} \operatorname{Meas}_{1}\left(\operatorname{Spec}_{\infty}[\mu]\right)=0 .
$$

The regions associated to the rotation numbers $r_{n}=\frac{1}{n}$ asymptotically remove all the area in $-2 \leq a \leq 2$.

For comparison with Theorem 2.1] we include the following result characterizing the set $\Omega_{B}$ of all parameter values for which all orbits are bounded, given in Beardon, Bullett and Rippon [2], based on results of Herman [8, VIII2.4].

Theorem 2.3 The set $\Omega_{B}$ consists of those parameter values $(\mu, \nu)$ for whose associated $(a, b)$ the map $T_{a b}$ is conjugate to a rotation of the plane. This occurs if and only if one of the following occur.

(1) $r\left(S_{a b}\right)$ is rational and $T_{a b}$ is of finite order, i.e. $T_{a b}^{(k)}=I$ for some finite $k$.

(2) $r\left(S_{a b}\right)$ is irrational, and $T_{a b}$ contains an invariant circle.

Examples given in part I and II show that cases (1) and (2) both occur. The set of parameter values for which case (1) occurs has Hausdorff dimension 1.

In $\S 4$ we study bounded solutions for the one-parameter "eigenvalue" families in which $\mu=$ constant. Recall that a Cantor set in $\mathbb{R}$ is a perfect ${ }^{1}$ totally disconnected set. The following theorem is the main result of the paper.

Theorem 2.4 For $\mu_{0} \in \mathbb{R}$ the set

$$
\operatorname{Spec}_{\infty}[\mu]:=\left\{E=2-\nu:(\mu, \nu) \in \Omega_{S B}\right\}
$$

is a Cantor set if $\mu$ is not in a countable exceptional set $\mathcal{E}$ that consists entirely of algebraic numbers.

The set $\mathcal{E}$ contains $\mu=0$, where $\operatorname{Spec}_{\infty}[0]=[0,4]$. As far as we know, this might be the only point in $\mathcal{E}$. If so, then $\Omega_{S B}$ would have the structure (Cantor set) $\times($ half-line) in the $(\mu, a)$-parameter space, in the region $-2 \leq a \leq 2$ and $\mu>0$. As indicated earlier, numerical evidence supports the assertion that $\operatorname{Spec}_{\infty}[\mu]$ has positive one-dimensional Lebesgue measure for all real values of $\mu$.

\section{Bounded Orbits}

We consider the set $\Omega_{S B}$ of parameter values having at least one bounded orbit.

Theorem 3.1 The set $\Omega_{S B}$ is a closed set. Each $(\mu, \nu) \in \Omega_{S B}$ the associated $T_{a b}$ possesses a bounded orbit $\mathcal{O}\left(\mathbf{v}_{0}\right)=\left\{\mathbf{v}_{n}: k \in \mathbb{Z}\right\}$ such that

$$
\left\|\mathbf{v}_{0}\right\|=\sup _{n \in \mathbb{Z}}\left\|T_{a b}^{(n)}\left(\mathbf{v}_{0}\right)\right\| .
$$

\footnotetext{
${ }^{1} \mathrm{~A}$ closed set $E$ is perfect if each $x \in E$ is a limit point of a infinite sequence of distinct points of $E$.
} 
Proof. Let $\left(a_{k}, b_{k}\right)$ be associated values to parameters $\left(\mu_{k}, \nu_{k}\right) \in \Omega_{S B}$ with $\left(a_{k}, b_{k}\right) \rightarrow(a, b)$. We can choose a bounded orbit $\mathcal{O}\left(\mathbf{v}_{0}^{(k)}\right)=\left\{\mathbf{v}_{n}^{(k)}: n \in \mathbb{Z}\right\}$ of $T_{a_{k} b_{k}}$ with

$$
\sup _{n \in \mathbb{Z}}\left\|\mathbf{v}_{n}^{(k)}\right\|=1
$$

and we may also suppose that

$$
1 \geq\left\|\mathbf{v}_{0}^{(k)}\right\| \geq 1-\frac{1}{k}
$$

by shifting the orbit appropriately. By compactness of the unit ball in $\mathbb{R}^{2}$ we may extract a subsequence so that

$$
\mathbf{v}_{0}^{\left(k_{j}\right)} \rightarrow \mathbf{v}_{0}
$$

and necessarily $\left\|\mathbf{v}_{0}\right\|=1$. Set $\mathbf{v}_{m}=T_{a b}^{(m)}\left(\mathbf{v}_{0}\right)$. Then, for each fixed $m \in \mathbb{Z}$,

$$
\mathbf{v}_{m}^{\left(k_{j}\right)} \rightarrow T_{a b}^{(m)}\left(\mathbf{v}_{0}\right)
$$

as $k_{j} \rightarrow \infty$, because $T_{a b}(\cdot)$ depends continuously on the parameters $(a, b) \in \mathbb{R}^{2}$. Since $\left\|\mathbf{v}_{m}^{\left(k_{j}\right)}\right\| \leq 1$, this yields

$$
\left\|\mathbf{v}_{m}\right\|=\left\|T_{a b}^{(m)}\left(\mathbf{v}_{0}\right)\right\| \leq 1 .
$$

Thus $T_{a b}$ has a bounded orbit, so $(a, b) \in \Omega_{S B}$, and furthermore this orbit attains its supremum

$$
\left\|\mathbf{v}_{0}\right\|=\sup _{m \in \mathbb{Z}}\left\|\mathbf{v}_{m}\right\|=1
$$

The argument applies to any $(a, b)$ associated to some $(\mu, \nu) \in \Omega_{S B}$ by taking all $\left(a_{k}, b_{k}\right)=(a, b)$, to give (3.1).

To prove Theorem 2.1 we establish the following preliminary result, using $(\mu, \nu)$-parameters.

Theorem 3.2 Let $\mu \in \mathbb{R}$ be fixed and let $\nu$ vary over $-\infty<\nu<\infty$.

(1) Let $0<r<\frac{1}{2}$ be rational. Then the set $I_{\mu}(r)$ of values $\nu$ such that $r\left(S_{\mu \nu}\right)=r$ is either a point $\nu^{ \pm}(r)$ or an interval $\left[\nu^{-}(r), \nu^{+}(r)\right]$. In the point case $T_{\mu \nu^{ \pm}}$is of finite order. In the interval case $T_{\mu \nu}$ is never of finite order, and $T_{\mu \nu}$ for $\nu \in\left[\nu^{-}(r), \nu^{+}(r)\right]$ contains a periodic orbit if and only if $\nu$ is one of the endpoints of the interval $\nu=\nu^{-}(r)$ or $\nu^{+}(r)$.

(2) Let $0<r<\frac{1}{2}$ be irrational. Then the set $I_{\mu}(r)$ of values $\nu$ such that $r\left(S_{\mu \nu}\right)=r$ consists of a point $\nu^{ \pm}(r)$.

(3) The set $I_{\mu}(0)$ of values $\nu$ with $r\left(S_{\mu \nu}\right)=0$ is a half-infinite interval $\left[\nu^{-}(0),+\infty\right)$. In this interval $T_{\mu \nu}$ is never periodic, and $T_{\mu \nu}$ has a periodic orbit only for $\nu=\nu^{-}(0)$.

(4) The set $I_{\mu}(1 / 2)$ of values $\nu$ with $r\left(S_{\mu \nu}\right)=\frac{1}{2}$ is a half-infinite interval $\left(-\infty, \nu^{+}\left(\frac{1}{2}\right)\right]$. In this interval $T_{\mu \nu}$ is never periodic, and $T_{\mu \nu}$ has a periodic orbit only for $\nu=\nu^{+}\left(\frac{1}{2}\right)$.

Proof. The continuity and nonincreasing properties of $S_{\mu \nu}$ in Theorem 2.2 of part I imply that for $0 \leq r \leq 1 / 2$ the set $\left\{\nu: r\left(S_{\mu \nu}\right)=r\right\}$ is either a point or an interval. We first consider cases (1), (3) and (4). Lemma 4.1 of part I implies that for rational $r\left(S_{\mu \nu}\right)=\frac{p}{q}$, the point case can occur if and only if $S_{\mu \nu}$ is periodic, and by Theorem 2.5 (iii) of part I, this occurs if and only if $T_{\mu \nu}$ is of finite order. Thus in the interval case $I(r)=\left[\nu^{-}(r), \nu^{+}(r)\right]$, the map $T_{\mu \nu}$ is never periodic, and the classification of Theorem 2.4 of part I showed that the circle map $S_{\mu \nu}$ 
then has either one or two periodic orbits. The proof of Theorem 2.4 of part I showed that in the interior of the rotation interval $\left(\nu^{-}(r), \nu^{+}(r)\right)$ the circle map $S_{\mu \nu}$ has two periodic orbits whose points alternate around the circle $0 \leq \theta \leq 2 \pi$. At the endpoint values $\nu^{+}(r), \nu^{-}(r)$ these coalesce into a single periodic orbit, which is case (i) of Theorem 2.4 of part I, and $T_{\mu \nu}$ then has a periodic orbit. This proves (1). Cases (3) and (4) follow similarly.

Now consider case (2), where $r\left(S_{\mu \nu_{0}}\right)=r$ is irrational. We show first that for any $\epsilon>0$ there exists $\nu^{\prime}$ in $\left[\nu_{0}, \nu_{0}+\epsilon\right]$ such that $S_{\mu \nu^{\prime}}$ has a periodic point, so that $r\left(S_{\mu \nu^{\prime}}\right)$ is rational. The proof of Lemma 4.1 of part I showed that

$$
\left.\frac{\partial}{\partial \nu} S_{\mu \nu}^{(2)}(\theta)\right|_{\nu=\nu_{0}}<0 .
$$

Thus, choosing $\nu_{1}=\nu_{0}+\epsilon$ there is a positive constant $\epsilon_{1}$ such that the lifted map $\tilde{S}_{\mu \nu}^{(2)}$ to the line has

$$
\tilde{S}_{\mu \nu_{1}}^{(2)}(\theta) \leq S_{\mu \nu_{0}}^{(2)}(\theta)-\epsilon_{1}, \quad 0 \leq \theta \leq 2 \pi
$$

since $S_{\mu \nu}^{(2)}$ is strictly monotone in $\theta$; this gives

$$
\tilde{S}_{\mu \nu_{1}}^{(2)}(\theta) \leq \tilde{S}_{\mu \nu_{0}}^{(2)}(\theta)=\epsilon_{1}, \quad \text { all } \quad \theta \in \mathbb{R}
$$

Since $R\left(S_{\mu \nu_{0}}\right)=r$ is irrational, one can find a positive integer $m$ such that

$$
0<S_{\mu \nu_{0}}^{(m)}(\theta)<\epsilon_{1} \quad(\bmod 2 \pi)
$$

Then as $\nu$ increases continuously in $\left[\nu_{0}, \nu_{0}+\epsilon\right]$, the endpoint $S_{\mu \nu_{0}}^{(m)}(\theta)$ moves continuously and monotonically downward, there exists some $v^{\prime} \in\left[\nu_{0}, \nu_{1}\right]$ with

$$
S_{\mu \nu}^{(m)}(\theta)=0 \quad(\bmod 2 \pi)
$$

by (3.2) the movement of $S_{\mu \nu}^{(m)}(\theta)$ as $\nu$ varies is counterclockwise by at least $\epsilon_{1}$. Now (3.4) shows that $S_{\mu \nu^{\prime}}$ has a periodic point of period $m$, hence $R\left(S_{\mu \nu^{\prime}}\right)$ is rational, as claimed. It follows that $r\left(S_{\mu \nu_{0}}\right)>r\left(S_{\mu \nu^{\prime}}\right)$ and $\nu_{0}<\nu^{\prime} \leq \nu_{0}+\epsilon$.

Next, a similar argument shows that for each $\epsilon>0$ there exists $\nu^{\prime \prime}$ in $\left[\nu_{0}-\epsilon, \nu_{0}\right]$ such that $S_{\mu \nu^{\prime \prime}}$ has a periodic point. Thus $r\left(S_{\mu \nu_{0}}\right)<r\left(S_{\mu \nu^{\prime \prime}}\right)$ with $\nu_{0}-\epsilon \leq \nu^{\prime \prime} \leq \nu_{0}$.

We conclude that the rotation interval $I(r)$ for the irrational rotation value $r$ (for fixed $\mu$ ) is contained in $\left[\nu_{0}-\epsilon, \nu_{0}+\epsilon\right]$ for all $\epsilon>0$, hence $I(r)=\left\{\nu_{0}\right\}$ is a point, which we label $\nu^{ \pm}(r)$.

Theorem 3.3 If the rotation number $r\left(S_{a b}\right)$ is irrational, then $T_{a b}$ has a bounded orbit.

Proof. We switch to $(\mu, \nu)$ parametrization, and suppose that $r\left(S_{\mu \nu_{0}}\right)=r$ is irrational, with $\mu=\frac{1}{2}(a-b), \nu_{0}=\frac{1}{2}(a+b)$; necessarily $0<r<\frac{1}{2}$. We consider $T_{\mu \nu}$ for variable $\nu$, and show we can pick a sequence $\left\{\nu_{k}: k \geq 1\right\}$ with $\nu_{k} \rightarrow \nu_{0}$ such that

(*) $T_{\mu \nu_{k}}$ has rational rotation number and a periodic orbit $\left\{\mathbf{v}_{n}^{(k)}: n \in \mathbb{Z}\right\}$, normalized with $\left\|\mathbf{v}_{0}^{(k)}\right\|=\max _{n \in \mathbb{Z}}\left\|\mathbf{v}_{n}^{(k)}\right\|=1$. 
To do this we pick a series of rational approximations $\left\{\frac{p_{k}}{q_{k}}\right\}$ approaching $r$ monotonically from above, and choose $\nu_{k}=\nu^{-}\left(\frac{p_{k}}{q_{k}}\right)$, for the rotation interval $I(r)=\left[\nu^{-}\left(\frac{p_{k}}{q_{k}}\right), \nu^{+}\left(\frac{p_{k}}{q_{k}}\right)\right]$. By Theorem 3.2 (1) $T_{\mu \nu_{k}}$ has a periodic orbit, which we can normalize by scaling to satisfy $(*)$. By compactness, we can extract a subsequence $\left\{\mathbf{v}_{0}^{(k)}\right\}$ such that $\mathbf{v}_{0}^{\left(k_{j}\right)} \rightarrow \mathbf{v}_{0}$ as $j \rightarrow \infty$. Then $\left\|\mathbf{v}_{0}\right\|=1$ and, just as in the proof of Theorem 3.1. $\mathcal{O}\left(\mathbf{v}_{0}\right)=\left\{\mathbf{v}_{n}=T_{\mu \nu_{0}}^{(n)}\left(\mathbf{v}_{0}\right): n \in \mathbb{Z}\right\}$ is a bounded orbit of $T_{\mu \nu_{0}}$, with

$$
\left\|\mathbf{v}_{0}\right\|=\max _{n \in \mathbb{Z}}\left\|\mathbf{v}_{n}\right\|=1
$$

as required.

We now deduce Theorems 2.1 and 2.2

Proof of Theorem 2.1, (1) Suppose that $r\left(S_{a b}\right)$ is rational. Theorem 2.4 (i) and (iii) of part I classify when $T_{a b}$ has a bounded orbit. All such orbits are periodic orbits of $T_{a b}$.

(2) The case that $r\left(S_{a b}\right)$ is irrational is covered by Theorem 3.3 .

Proof of Theorem 2.2. The excluded regions in the theorem, for $-2 \leq a \leq 0$ correspond to rotation number $\frac{1}{2}$ and for $a=2 \cos \theta \geq 0$ and $\frac{\pi}{n+1}<\theta<\frac{\pi}{n}$ for $n \geq 2$ they correspond to rotation number $\frac{1}{n+1}$. The proof of Theorem 3.3 of part I shows that for $a \geq b$ (that is, $\mu \geq 0$ ) and parameter values outside the stated range $\mathcal{R}, S_{\mu \nu}$ has an associated matrix $M$ with $\operatorname{Tr}(M)>2$, and has two periodic orbits, corresponding to the two real eigenvectors of $M$. Case (2) of Theorem 2.4 then yields that every orbit of $T_{\mu \nu}$ is unbounded.

The allowed region $\mathcal{R}$ in Theorem 2.2 containing $\Omega_{S B}$ was pictured in $\S 2$, in Figure 2.1] It can be compared with Figure 3.1 below where we numerically plot the rotation number $r\left(T_{a b}\right)$ in the $(\mu, a)$-parameter plane, where $\mu \geq 0$ and $-2 \leq a \leq 2$, in the range $0 \leq \mu \leq 2$.

The rotation intervals for $r=\frac{1}{2}, \frac{2}{5}, \frac{1}{3}, \frac{2}{7}, \frac{1}{4}, \frac{1}{5}$ and $\frac{1}{6}$ are visible in Figure 3.1] We note that the rotation interval with $r=1 / 3$ pictured above is larger than that excluded from the region $\mathcal{R}$, for Theorem 2.1 excludes no values for $0 \leq \mu \leq 1$ with $0 \leq \alpha<1$, corresponding to $r=\frac{1}{3}$.

We now describe numerical evidence suggesting that $\Omega_{S B}$ has positive Lebesgue measure, in the region $0 \leq \mu \leq 1$. The question is equivalent to: Does the (open) set consisting of the interiors of all rational rotation intervals have full Lebesgue measure in the region $-2 \leq a \leq 2$, $0 \leq \mu \leq 1$ ? We suggest it does not. Consider, for fixed $\mu$, the amount of area in $\{-2 \leq a \leq 2\}$ left uncovered by the interiors of rational rotation intervals. At $\mu=0$ the full interval $[-2,2]$ of measure 4 is left uncovered. (For $\mu=0$ all rotation intervals have width 0 , by Example 3.1 of part I.) We observe empirically in Figure 3.1 that the width of rational rotation numbers $r=\frac{1}{n}$ appears to be nondecreasing in $\mu$. We know from Example 3.3 of part $\mathrm{I}$ that for certain values, such as $r=\frac{3}{8}$, the rotation interval has width zero for $0 \leq \mu \leq \infty$. There are rational rotation numbers (e.g. $r=\frac{2}{9}$ ) where the length of the rotation interval as a function of $\mu$ is sometimes increasing and sometimes decreasing. For small $\mu$ we found empirically that the total uncovered length appears nonincreasing as a function of $\mu$. If this holds up to $\mu=1$, then in the range $0 \leq \mu \leq 1$, the uncovered length would be minimized at $\mu=1$. For $\mu=1$ we numerically estimated the length in $\{-2 \leq a \leq 2\}$ left uncovered by rotation intervals $r=\frac{p}{q}$ with denominator $q \leq N$ and obtained the data in the following table. 


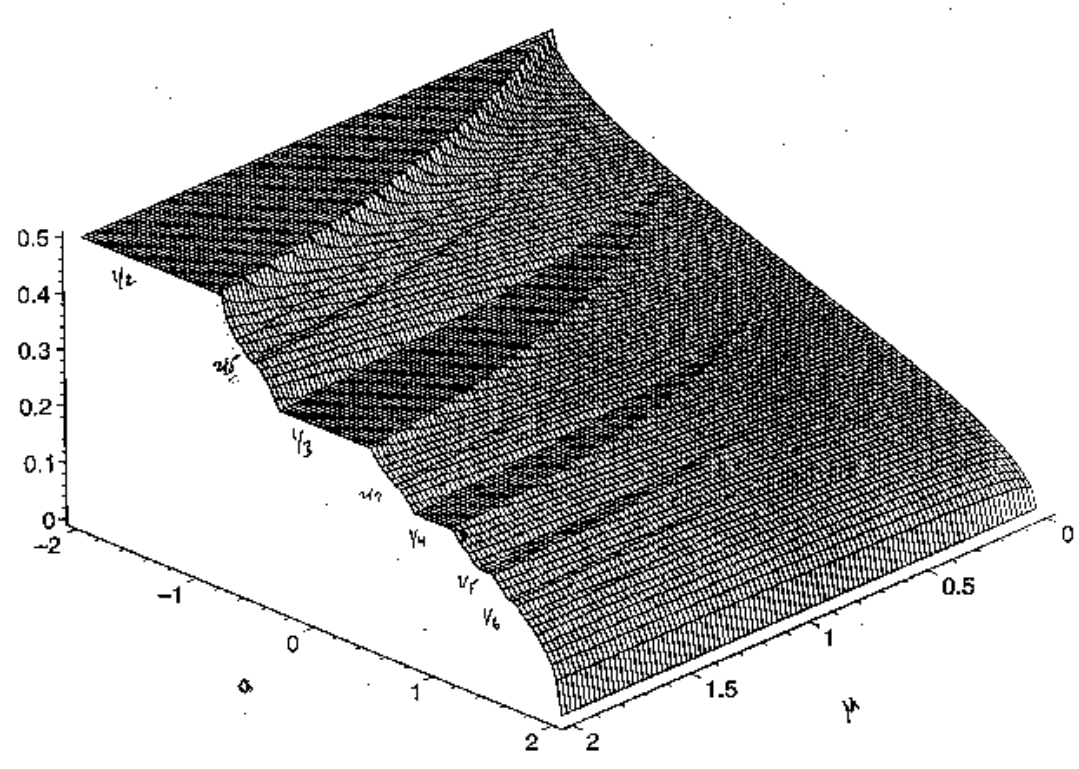

Figure 3.1: Rotation number in $(\mu, a)$-plane, $0 \leq \mu \leq 2,-2 \leq a \leq 2$.

This data suggests that in the limit as the denominator cutoff $N \rightarrow \infty$, for $\mu=1$ there is an uncovered set of positive Lebesgue measure, and this measure is approximately 2 . Under the monotonicity hypothesis above, the two-dimensional Lebesgue measure of $\Omega_{S B}$ in the region $0 \leq \mu \leq 1$ in the $(\mu, a)$ plane would lie between 2 and 4 .

The numerical evidence above led us to formulate Conjecture B. As additional evidence in favor of Conjecture B, we numerically located orbits that appear to be invariant circles, described in $\S 5$ of part I. If invariant circles were a measure zero phenomenon, we would not expect to numerically find such invariant circles. It appears plausible that $\Omega_{B}$ may also have positive (two-dimensional) Lebesgue measure.

We now prove Theorem 2.3, which amounts to the equality $\Omega_{R}=\Omega_{B}$, where $\Omega_{R}$ are the

\begin{tabular}{|l|c|}
\hline Denominator & Area \\
\hline $\mathrm{N}=1$ & 4.00000000 \\
$\mathrm{~N}=2$ & 3.23606798 \\
$\mathrm{~N}=4$ & 2.45423817 \\
$\mathrm{~N}=8$ & 2.17951560 \\
$\mathrm{~N}=16$ & 2.09869137 \\
$\mathrm{~N}=32$ & 2.05071812 \\
$\mathrm{~N}=64$ & 2.02839432 \\
$\mathrm{~N}=128$ & 2.01690968 \\
$\mathrm{~N}=256$ & 2.01094576 \\
\hline
\end{tabular}

Table 3.1: Uncovered length: case $\mu=1$. 
parameter values for which $T_{a b}$ is conjugate to a rotation of the plane. We follow Beardon, Bullett and Rippon [2.

Proof of Theorem 2.3. Case (1) is covered by Theorem 2.4 of part I. Case (2) follows from results of Herman [8, VIII.2.4]. For $S_{a b}$ having irrational rotation number he establishes the dichotomy that either $T_{a b}$ has an invariant circle, or else it is topologically transitive, i.e. it contains an orbit that is dense in $\mathbb{R}^{2}$. Such an orbit is unbounded.

If $T_{a b}$ is topologically conjugate to a rotation of the plane, then all orbits are bounded. The fact that $T_{a b}$ is topologically conjugate to a rotation in cases (1) and (2) follows also from results of Herman [8, VIII.2.5], which uses facts from Herman [7.

Finally we consider the possible existence of localized orbits. We call an orbit $\left\{\mathbf{x}_{n}: n \in \mathbb{Z}\right\}$ weakly localized if

$$
\lim _{n \rightarrow \pm \infty}\left\|\mathbf{x}_{n}\right\|=0 \text {. }
$$

Theorem 2.3 implies that if $T_{\mu \nu}$ had a localized orbit, then necessarily $S_{\mu \nu}$ has irrational rotation number, and $T_{\mu \nu}$ is not conjugate to a rotation. It is not known whether any $T_{\mu \nu}$ with the latter property exists. Herman [8] observed that any such map necessarily has a dense orbit. Herman [8. VIII.2.4] conjectured and gave evidence for the existence of some element of the Froeschlé group having this property. Here the Froeschlé group is the group of homeomorphisms of the plane generated by $S L(2, \mathbb{R})$ and all maps

$$
G_{a b}=\left[\begin{array}{cc}
1 & 0 \\
F_{a b}(x) & 1
\end{array}\right]
$$

for real $a, b$, viewed as acting on column vectors. Bedford, Bullett and Rippon [2, pp. 673-674] discuss the possibility that this occurs for some maps $T_{a b}$, and suggest it might even occur somewhere on the parameter line $\nu=1 / 2(a+b)=0$.

\section{Cantor Set Spectra: Constant $\mu$}

We consider the family $T_{\mu \nu}$ for fixed $\mu$ and variable $\nu$. Recall that for these families, one is particularly interested in knowing for which $E=2-\nu$ one has bounded orbits; these values of $E$ correspond to eigenvalues of the nonlinear Schrödinger system (1.8) giving extended states or localized states. For the case $\mu=0$ where the maps $T_{\mu \nu}$ are linear we have:

Theorem 4.1 For $\mu=0, T_{\mu \nu}$ has a nontrivial bounded orbit if and only if $\nu \in[-2,2]$; all orbits are bounded if and only if $\nu \in(-2,2)$.

Proof. If $\nu \notin[-2,2]$, then, by Theorem 2.1] $T_{\mu \nu}$ has no bounded orbits. If $\nu= \pm 2$, then explicit calculation verifies that $S_{\mu \nu}$ has exactly one periodic orbit; by Theorem 2.4 (i) of part I, then $T_{\mu \nu}$ has exactly one nontrivial bounded orbit. The remaining case, $\nu \in(-2,2)$, was covered in Example 3.1 of part I which showed it is conjugate to a rotation. In this case $T_{\mu \nu}$ either is periodic or else has an invariant circle (an ellipse, in fact). 
For fixed $\mu \in \mathbb{R}$ say that a rational rotation interval $I_{\mu}(r)$ in the $\nu$-variable is nondegenerate if $I_{\mu}(r)=\left[\mu_{\mu}^{-}(r), \nu_{\mu}^{+}(r)\right]$, has positive measure and is degenerate if $I_{\mu}(r)=\left[\nu_{\mu}^{0}(r)\right]$ is a point. We let

$$
E\left(\frac{m}{n}\right):=\left\{\mu \geq 0: I_{\mu}\left(\frac{m}{n}\right) \text { is degenerate }\right\} .
$$

denote the "'exceptional" set where degeneracy occurs.

To show that $\operatorname{Spec}_{\infty}[\mu]:=\left\{E=2-\nu: T_{\mu \nu}\right.$ has a bounded orbit $\}$ is a Cantor set for a given $\mu$, we must show that it is totally disconnected; for this it is necessary and sufficient that there are a dense set of rational rotation numbers in $[0,1 / 2]$ whose rotation intervals at $\mu$ are nondegenerate. The following result gives a criterion for nondegeneracy which applies to rotation numbers having an odd prime denominator.

Theorem 4.2 Let $q \geq 3$ be prime. For each rational $r=\frac{j}{q}$ with $\left.1 \leq j \leq \frac{q-1}{2}\right\}$ the set $E\left(\frac{j}{q}\right)$ of all $\mu \geq 0$ for which the rotation interval of rotation number $\frac{j}{q}$ is degenerate is a finite set of algebraic numbers.

Remark. Each set $E\left(\frac{j}{q}\right)$ includes $\mu=0$, and may include other points. For example, $E\left(\frac{2}{7}\right)$ contains $\mu=\frac{1}{2}$. Theorem 4.2 does not generalize to hold for all $E\left(\frac{m}{n}\right)$; indeed Example 3.3 of part I shows that $E\left(\frac{1}{8}\right)$ is the positive real line.

To prove this result we first establish some preliminary lemmas. We shall describe iteration of the map $T_{a b}$ assuming the iterates have a given symbol sequnece $\mathcal{S}=\left(S_{1}, S_{2}, \ldots, S_{n}\right)$, with $S_{i}= \pm 1$ instructing whether $F_{a b}(x)=a x$ or $b x$. We keep track of the iterates for a symbol sequence using $(a, \mu)$ coordinates, where $a$ and $\mu$ are viewed as indeterminates; note that $b=a-2 \mu$. Requiring periodicity of a particular orbit then gives polynomial equations that $a$ and $\mu$ must satisfy. We first treat the case corresponding to $\mu \neq 0$.

Lemma 4.1 Given a symbol sequence $\mathcal{S}=\left(S_{1}, \ldots, S_{n}\right) \in\{1,-1\}^{n}$ define bivariate polynomials $p_{n}^{\mathcal{S}}(a, \mu) \in \mathbb{Z}[a, \mu]$ by

$$
\left[\begin{array}{c}
p_{n}^{\mathcal{S}}(a, \mu) \\
\left.p_{n-1}^{\mathcal{S}}(a, \mu)\right)
\end{array}\right]:=\left[\begin{array}{cc}
F_{a b}\left(S_{n-1}\right) & -1 \\
1 & 0
\end{array}\right] \cdots\left[\begin{array}{cc}
F_{a b}\left(S_{1}\right) & -1 \\
1 & 0
\end{array}\right]\left[\begin{array}{l}
1 \\
0
\end{array}\right]
$$

where

$$
F_{a b}\left(S_{i}\right)=\left\{\begin{array}{ccc}
a & \text { if } \quad S_{i}=1 \\
a-2 \mu & \text { if } \quad S_{i}=-1
\end{array}\right.
$$

Now suppose $\mu \neq 0$ is a fixed real number, and view each $p_{n}^{\mathcal{S}}(a, \mu)$ as a univariate polynomial in $\mathbb{R}[a]$. If $\mathcal{S}, \mathcal{S}^{\prime}$ are two distinct symbol sequences, then

$$
\left(p_{n}^{\mathcal{S}}(a, \mu), p_{n-1}^{\mathcal{S}}(a, \mu)\right) \not \equiv\left(p_{n}^{\mathcal{S}^{\prime}}(a, \mu), p_{n-1}^{\mathcal{S}^{\prime}}(a, \mu)\right) .
$$


Proof. It suffices to show that, given $\mu \neq 0$, the sequence $\mathcal{S}$ is uniquely reconstructible from the data $\left(p_{n}(a, \mu), p_{n-1}(a, \mu)\right):=\left(p_{n}^{\mathcal{S}}(a, \mu), p_{n-1}^{\mathcal{S}}(a, \mu)\right)$. We define

$$
\left(p_{1}^{\mathcal{S}}(a, \mu), p_{0}^{\mathcal{S}}(a, \mu)\right):=(1,0)
$$

and we have, for $1 \leq j \leq n$,

$$
\left[\begin{array}{c}
p_{j}^{\mathcal{S}}(a, \mu) \\
p_{j-1}^{\mathcal{S}}(a, \mu)
\end{array}\right]=\left[\begin{array}{cc}
F_{a b}\left(S_{j}\right) & -1 \\
1 & 0
\end{array}\right]\left[\begin{array}{c}
p_{j-1}^{\mathcal{S}}(a, \mu) \\
p_{j-2}^{\mathcal{S}}(a, \mu)
\end{array}\right] .
$$

By induction $j \geq 1, p_{j}^{\mathcal{S}}(a, \mu) \in \mathbb{R}[a]$ is a monic polynomial of degree $j-1$. Applying (4.5), given $j=n$, the given data $\left(p_{n}(a, \mu), p_{n-1}(a, \mu)\right)$ are monic polynomials of degree $n, n-1$ respectively. Now there is a unique choice of symbol $S_{n}$ such that

$$
p_{n-2}(a, \mu):=F_{a b}\left(S_{n}\right) p_{n-1}(a, \mu)-p_{n}(a, \mu)
$$

is a polynomial in $a$ of degree at most $n-2$. Thus $S_{n}$ is uniquely determined, so we can calculate $\left(p_{n-1}^{S}(a, \mu), p_{n-2}^{S}(a, \mu)\right)$. This process can now be repeated to successively determine $S_{n-1}, S_{n-2}, \ldots, S_{2}, S_{1}$.

We next treat the case corresponding to $\mu=0$, where we obtain univariate polynomials.

Lemma 4.2 Let the univariate polynomials $P_{n}(a) \in \mathbb{Z}[a]$ for $n \in \mathbb{Z}$ be given by the recurrence

$$
p_{n}(a)=a p_{n-1}(a)-p_{n-2}(a)
$$

with the initial conditions $p_{0}(a)=0, p_{1}(a)=1$. Then $p_{-n}(a)=-p_{n}(a)$ and, for $n \geq 2$,

$$
p_{n}(a)=\prod_{j=1}^{n-1}\left(a-\cos \frac{\pi j}{n}\right) .
$$

For $n=q \geq 3$ a prime, the irreducible factorization of $p_{q}(a)$ over $\mathbb{Q}[a]$ is

$$
p_{q}(a)=(-1)^{\frac{n-1}{2}} p_{q}^{(0)}(a) p_{q}^{(0)}(-a)
$$

where

$$
p_{q}^{(0)}(a)=\prod_{j=1}^{\frac{q-1}{2}}\left(a-\cos \frac{\pi j}{q}\right) \in \mathbb{Z}[a]
$$

has degree $\frac{q-1}{2}$.

Proof. The relation $p_{-n}(a)=p_{n}(a)$ is easily checked by induction on $n \geq 1$. For $n=1,2$ we have

$$
p_{n}\left(x+\frac{1}{x}\right)=\frac{x^{n}-\left(\frac{1}{x}\right)^{n}}{x-\frac{1}{x}},
$$


and this relation holds for all $n \geq 3$, by induction on $n$, verifying the recurrence (4.6). Thus

$$
p_{n}\left(x+\frac{1}{x}\right)=x^{1-n} \frac{x^{2 n}-1}{x^{2}-1} .
$$

The right side of (4.10) clearly has zeros at $x=e^{\frac{\pi i j}{n}}$ for $1 \leq j \leq n-1$ and $n+1 \leq j \leq 2 n-1$. Now

$$
a=x+\frac{1}{x}=e^{\frac{\pi i j}{n}}+e^{-\frac{\pi i j}{n}}=2 \cos \frac{\pi j}{n},
$$

and these take $n-1$ distinct values for $1 \leq j \leq n-1$ (repeated for $n+1 \leq j \leq 2 n$ ). This accounts for $n-1$ distinct roots of the polynomial $p_{n}(a)$, and since it is a monic polynomial of degree $n-1$, the factorization (4.7) follows. In fact $p_{n}(x)=U_{n}\left(\frac{1}{2} a\right)$, where $U_{n}(a)$ is the Chebyshev polynomial of the second kind, cf. Rivlin [17, Chap. 5].

Now suppose $n=q \geq 3$ is an odd prime. Then we have $\cos \frac{\pi(q-j)}{q}=-\cos \frac{\pi j}{q}$, which yields the factorization (4.8), (4.9) over $\mathbb{Q}[a]$. Finally $p_{q}^{(0)}(a) \in \mathbb{Z}[a]$ is irreducible because its roots are a complete set of Galois conjugates in $\mathbb{Q}\left(\zeta_{q}+\zeta_{q}^{-1}\right)$, with $\zeta_{q}=\exp \left(\frac{2 \pi i}{q}\right)$, which is a field of degree $\frac{q-1}{2}$ over $\mathbb{Q}$.

Proof of Theorem 4.2. By Theorem 3.2(i) a rational rotation interval $I_{\mu}\left(\frac{m}{n}\right)$ is degenerate if and only if the corresponding $T_{\mu \nu}$ is periodic. By Theorem 2.3 of part I this occurs if and only if $(0,1)$ is a periodic point, with

$$
T_{\mu \nu}^{(n)}(0,1)=(0,1)
$$

Since $T_{\mu \nu}(0,1)=(-1,0)$ we can write this condition as

$$
\left[\begin{array}{cc}
F_{a b}\left(S_{n-1}\right) & -1 \\
1 & 0
\end{array}\right] \cdots\left[\begin{array}{cc}
F_{a b}\left(S_{1}\right) & -1 \\
1 & 0
\end{array}\right]\left[\begin{array}{c}
-1 \\
0
\end{array}\right]=\left[\begin{array}{l}
0 \\
1
\end{array}\right],
$$

where $\mathcal{S}:=\left(S_{1}, S_{2}, \ldots, S_{n}\right)$ is a certain symbol sequence with each $S_{i}= \pm 1$, and we define

$$
F_{a b}\left(S_{i}\right):= \begin{cases}a & \text { if } \quad S_{i}=1 \\ b=a-2 \mu & \text { if } \quad S_{i}=-1\end{cases}
$$

We define

$$
\left(p_{1}^{\mathcal{S}}(a, \mu), p_{0}^{\mathcal{S}}(a, \mu)\right)=(1,0)
$$

and set

$$
-\left[\begin{array}{c}
p_{j}^{\mathcal{S}}(a, \mu) \\
p_{j-1}^{\mathcal{S}}(a, \mu)
\end{array}\right]=\left[\begin{array}{cc}
F_{a b}\left(S_{j}\right) & -1 \\
1 & 0
\end{array}\right] \cdots\left[\begin{array}{cc}
F_{a b}\left(S_{1}\right) & -1 \\
1 & 0
\end{array}\right]\left[\begin{array}{c}
-1 \\
0
\end{array}\right] .
$$

The polynomials $p_{j}^{\mathcal{S}}(a, \mu)$ satisfy the recursion

$$
p_{j}^{\mathcal{S}}(a, \mu):=F_{a b}\left(S_{j}\right) p_{j-1}^{\mathcal{S}}(a, \mu)-p_{j-1}^{\mathcal{S}}(a, \mu)
$$

which was studied in Lemma 4.1. For $\mu=0, F_{\mu \nu}(S) \equiv a$ and the recurrence is independent of the symbol sequence $\mathcal{S}$, and $p_{j}^{S}(a, 0)=p_{j}(a)$, the polynomials in Lemma 4.2. The definition (4.12) for $j=n$ put in (4.11) becomes

$$
-\left(p_{n}^{\mathcal{S}}(a, \mu), p_{n-1}^{\mathcal{S}}(a, \mu)\right)=(0,1)
$$


so that, viewing $\mu \in \mathbb{R}$ as fixed, the two polynomials

$$
p_{n}^{\mathcal{S}}(a, \mu)=0 \quad \text { and } \quad p_{n-1}^{\mathcal{S}}(a, \mu)+1=0
$$

have a common root in the $a$-variable. For a symbol sequence $\mathcal{S}$ corresponding to the rotation interval $I_{\mu}\left(\frac{m}{n}\right)$, a common root is $a$ is a necessary and sufficient condition for degeneracy.

We now study the common root condition for an arbitrary symbol sequence $\mathcal{S}=\left(S_{1}, S_{2}, \ldots, S_{n-1}\right)$. We view $\mu$ as a second indeterminate so that $p_{n}^{\mathcal{S}}(a, \mu)$ is a bivariate polynomial in $\mathbb{Z}[a, \mu]$. Consider the resultant

$$
R_{n}^{\mathcal{S}}(\mu):=\operatorname{Res}_{a}\left[p_{n}^{\mathcal{S}}(a, \mu), p_{n-1}^{\mathcal{S}}(a, \mu)-1\right] .
$$

It is immediate that $R_{n}^{\mathcal{S}}(\mu) \in \mathbb{Z}[\mu]$, using the determinant formula for the resultant. A common root occurs at $\mu=\mu_{0}$ if the resultant vanishes at $\mu_{0}$. There are two cases.

Case 1. $R_{n}^{\mathcal{S}}(\mu)$ is not identically 0.

Since $R_{n}^{\mathcal{S}}(\mu) \in \mathbb{Z}[\mu]$, all roots of $R_{n}^{\mathcal{S}}(\mu)$ are algebraic numbers. Also $R_{n}^{\mathcal{S}}(\mu)$ is of degree at most $2 n-1$, so there are at most $2 n-1$ such roots. For all $\mu$ not in this set, the equations (4.14) didn't have a common root.

Case 2. $R_{n}^{S}(\mu)$ is identically 0.

In this case the two polynomials in (4.14) have a common bivariate factor $q(a, \mu)$, and we have

$$
p_{n}^{\mathcal{S}}(a, \mu)=q(a, \mu) \tilde{q}(a, \mu)
$$

in which $1 \leq \operatorname{deg}_{a}(q(a, \mu)) \leq n-1$. By Gauss' lemma $q(a, \mu) \in \mathbb{Z}[a, \mu]$, and is a monic polynomial (since $p_{n}^{S}(a, \mu)$ is monic).

We now suppose that $n=q \geq 3$ is prime. The proof proceeds in three steps, which we will subsequently establish.

Step 1. The polynomials $q(a, \mu)$ and $\tilde{q}(a, \mu)$ are necessarily monic polynomials in $\mathbb{Z}[a, \mu]$ of degree $\frac{p-1}{2}$ in $a$.

Step 2. A nontrivial factorization (4.15) over $\mathbb{Z}[a, \mu]$ exists if and only if the sequence $\mathcal{S}=$ $\left(S_{1}, S_{2}, \ldots, S_{p-1}\right)$ is a palindrome, i.e. $S_{j}=S_{p-j}$ for $1 \leq j \leq \frac{p-1}{2}$.

Step 3. For any degenerate rotation interval $I_{\mu}\left(\frac{k}{p}\right), 1 \leq k \leq p-1$, its unique legal symbol sequence $\mathcal{S}$ has $S_{1}=-1$ and $S_{p-1}=1$, so $\mathcal{S}$ is not a palindrome.

Step 1 follows using Lemma 4.2, Given the bivariate factorization (4.15) over $\mathbb{Z}[a, \mu]$, setting $\mu=0$ gives

$$
p_{q}(a)=q(a, 0) \tilde{q}(a, 0)
$$


Now $\operatorname{deg}_{a}(q(a, 0))=\operatorname{deg}_{a}(q(a, \mu))$ because $q(a, \mu)$ is a monic polynomial in $a$; similarly for $\tilde{q}(a, 0)$. Lemma 4.2 says that $p_{q}(a)$ factors over $\mathbb{Z}(a)$ into two irreducible factors of degree $\frac{q-1}{2}$, necessarily $q(a, 0)$ and $\tilde{q}(a, 0)$ must be these two factors. We conclude that

$$
\operatorname{deg}_{a}(q(a, \mu))=\operatorname{deg}_{a}(\tilde{q}(a, \mu))=\frac{q-1}{2}
$$

completing step 1 .

Step 2 will follow using Lemma 4.1. For an arbitrary $n \geq 1$ we can apply the Euclidean algorithm. We write

$$
\left(p_{n}^{\mathcal{S}}(a, \mu), p_{n-1}^{\mathcal{S}}(a, \mu)+1\right)=\left(p_{n}^{\mathcal{S}}(a, \mu)+p_{0}^{\mathcal{S}^{R}}(a, \mu), p_{n-1}^{\mathcal{S}}(a, \mu)+p_{1}^{\mathcal{S}^{R}}(a, \mu)\right),
$$

in which $\mathcal{S}^{R}$ is the reversed sequence

$$
\mathcal{S}^{R}:=\left(S_{n-1}, S_{n-2}, \ldots, S_{1}\right)
$$

Now the recursion (4.13) for $\mathcal{S}$ and $\mathcal{S}^{R}$ gives

$$
\begin{gathered}
\left(p_{n-j}^{\mathcal{S}}(a, \mu)+p_{j}^{\mathcal{S}^{R}}(a, \mu)\right)-F_{a b}\left(\mathcal{S}_{n-j}\right)\left(p_{n-j-1}^{\mathcal{S}}(a, \mu)+p_{j+1}^{\mathcal{S}^{R}}(a, \mu)\right) \\
=-\left(p_{n-j-2}^{\mathcal{S}}(a, \mu)+p_{j+2}^{\mathcal{S}^{R}}(a, \mu)\right) .
\end{gathered}
$$

We obtain, by induction on $j \geq 1$, that

$$
\begin{aligned}
G(a, \mu) & =\text { g.c.d. }\left(p_{n}^{\mathcal{S}}(a, \mu), p_{n-1}^{\mathcal{S}}(a, \mu)-1\right) \\
& =\text { g.c.d. }\left(p_{n-j}^{\mathcal{S}}(a, \mu)+p_{j}^{\mathcal{S}^{R}}(a, \mu), p_{n-j-1}^{\mathcal{S}}(a, \mu)+p_{j+1}^{\mathcal{S}^{R}}(a, \mu) .\right.
\end{aligned}
$$

Now suppose $n=2 m+1$ and choose $j=\frac{m-1}{2}$. Then the right side terms are

$$
\begin{aligned}
& r_{1}^{\mathcal{S}}(a, \mu):=p_{\frac{n+1}{2}}^{\mathcal{S}}(a, \mu)+p_{\frac{n-1}{2}}^{S^{4}}(a, \mu) \\
& r_{2}^{\mathcal{S}}(a, \mu)=p_{\frac{n-1}{2}}^{\mathcal{S}}(a, \mu)+p_{\frac{n+1}{2}}^{\mathcal{S}^{R}}(a, \mu) .
\end{aligned}
$$

These polynomials lie in $\mathbb{Z}[a, \mu]$, and in the $a$-variable they are both of degree $\frac{n-1}{2}$, with monic top degree term $a^{\frac{n-1}{2}}$. Thus $r^{S}(a, \mu)=r_{1}^{\mathcal{S}}(a, \mu)-r_{2}^{\mathcal{S}}(a, \mu) \operatorname{has} \operatorname{deg}_{a}\left(r^{\mathcal{S}}(a, \mu)\right)<\frac{n-1}{2}$.

Now we impose the stronger condition that $n=q \geq 3$ is prime. There are two subcases.

Subcase 1. $r^{\mathcal{S}}(a, \mu) \not \equiv 0$.

Now $G(a, \mu)$ divides $r^{\mathcal{S}}(a, \mu)$ hence we conclude

$$
\operatorname{deg}_{a}(G(a, \mu))<\frac{q-1}{2} .
$$

But by step 1, any nontrivial divisor of $p_{n}^{\mathcal{S}}(a, \mu)$ has degree at least $\frac{q-1}{2}$, a contradiction. Thus Subcase 1 never occurs. 
Subcase 2. $r^{\mathcal{S}}(a, \mu) \equiv 0$.

We now have

$$
r^{\mathcal{S}}(a, \mu)=\left(p_{\frac{q+1}{2}}^{\mathcal{S}}(a, \mu)-p_{\frac{q+1}{2}}^{\mathcal{S}^{R}}(a, \mu)+\left(p_{\frac{q-1}{2}}^{\mathcal{S}}(a, \mu)-p_{\frac{q-1}{2}}^{\mathcal{S}^{R}}(a, \mu)\right)=0\right.
$$

We assert that this equation implies the separate equalities

$$
\begin{aligned}
& p_{\frac{q+1}{2}}^{\mathcal{S}}(a, \mu)=p_{\frac{q+1}{2}}^{\mathcal{S}^{R}}(a, \mu) \\
& p_{\frac{q-1}{2}}^{\mathcal{S}}(a, \mu)=p_{\frac{q-1}{2}}^{\mathcal{S}^{R}}(a, \mu) .
\end{aligned}
$$

Indeed, considering these polynomials in $\mathbb{Z}[a, \mu]$, the monomials in (4.18) must cancel term-byterm. However every monomial appearing in $p_{j}^{\mathcal{S}}(a, \mu)$ for $j \geq 1$ has its total degree congruent to $j-1(\bmod 2)$, as one proves by induction on $j \geq 1$. Thus each monomial appearing in

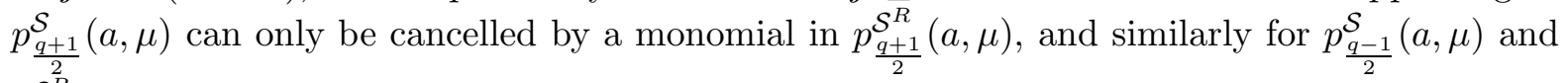
$p_{\frac{\mathcal{q}^{R}}{2}}^{\frac{\mathcal{S}^{2}}{2}}(a, \mu)$. Thus (4.19) follows.

We next apply Lemma 4.1 with $n=\frac{q+1}{2}$ to (4.19), to conclude that $\mathcal{S}=\mathcal{S}^{R}$, so the sequence $\mathcal{S}$ must be a palindrome. Conversely, if $\mathcal{S}$ is a palindrome, then (4.19) holds, whence $r^{\mathcal{S}}(a, \mu)=0$, so that

$$
q(a, \mu):=\text { g.c.d. }\left(p_{q}^{\mathcal{S}}(a, \mu), p_{q-1}^{\mathcal{S}}(a, \mu)-1\right)=p_{\frac{q+1}{2}}^{\mathcal{S}}(a, \mu)-p_{\frac{q-1}{2}}^{\mathcal{S}}(a, \mu)
$$

is a nontrivial factor satisfying (4.15). This completes step 2 .

Step 3 is a calculation. For a degenerate rotation interval we must have $T_{\mu v_{0}}^{(q)}(0,1)=(0,1)$. The sign $S_{1}$ is associated to $T_{\mu v_{0}}(-1,0)$, hence $S_{1}=-1$. The preimage $T_{\mu v_{0}}^{(-1)}(0,1)$ necessarily has the form $(1, *)$, hence $S_{q-1}=+1$. Thus the associated symbol sequence $\mathcal{S}$ is not a palindrome. This completes step 3 .

We conclude from steps 1-3 combined that Case 2 can never occur for any degenerate rotation interval $I_{\mu}\left(\frac{j}{q}\right)$ where $q \geq 3$ is prime and $1 \leq j \leq \frac{q-1}{2}$.

To complete the proof of Theorem 4.2] suppose that $\mu$ is a value such that $I_{\mu}\left(\frac{j}{q}\right)$ is degenerate. Then $\mu$ must be a value allowed by Case 1 for some symbol sequence $\mathcal{S}=\left(S_{1}, \ldots, S_{q-1}\right)$. There are at most $2^{q-1}$ such sequences, each of which has at most $2 q-1$ allowed values of $\mu$, all algebraic numbers. Thus $E\left(\frac{j}{q}\right)$ contains at most $2^{q-1}(2 q-1)$ elements.

We are now in a position to establish that $\operatorname{Spec}_{\infty}[\mu]$ is a Cantor set whenever $\mu$ is a transcendental number.

Proof of Theorem 2.4. Let $\mu \in \mathbb{R}$ be fixed. We show $\operatorname{Spec}_{\infty}[\mu]$ is totally disconnected whenever $\mu \in \mathbb{R}$ is transcendental. The set $\operatorname{Spec}_{\infty}[\mu]$ is closed, since it is the intersection of the closed sets $\{(\mu, \nu):-\infty<\nu<\infty\}$ and $\Omega_{S B}$. To show it is totally disconnected it suffices to show that between any two points $\nu_{0}, \nu_{1} \in \operatorname{Spec}_{\infty}[\mu]$ there is an open interval in its complement. If $\nu_{0}, \nu_{1}$ have the same rotation number they are necessarily the endpoints of a nontrivial rational rotation interval $I_{\mu_{0}}(r)$ whose interior is in the complement of $\operatorname{Spec}_{\infty}[\mu]$. If 
the rotation numbers are unequal, say $r\left(S_{\mu_{0} \nu_{0}}\right)<r\left(S_{\mu_{1} \nu_{1}}\right)$ then one can find a rational $\frac{j}{q}$ with $q$ prime such that

$$
r\left(S_{\mu_{0} \nu_{0}}\right)<\frac{j}{q}<r\left(S_{\mu_{1} \nu_{1}}\right)
$$

Theorem 4.2 guarantees that the rotation interval $I_{\mu}\left(\frac{j}{q}\right)$ is nontrivial, and by Theorem 2.4 of part I its interior is not in $\Omega_{S B}$. Thus $\operatorname{Spec}_{\infty}[\mu]$ is a totally disconnected set.

The set $\operatorname{Spec}_{\infty}[\mu]$ is a perfect set for all $\mu \in \mathbb{R}$. Indeed, given $\nu \in \operatorname{Spec}_{\infty}[\mu]$, the rotation number $r\left(S_{\mu \nu}\right)$ can be approximated both from above and below by sequences $\left(\mu, \nu_{n}^{+}\right),\left(\mu, \nu_{n}^{-}\right) \in$ $\Omega_{O B}$ having irrational rotation numbers converging to $r\left(S_{\mu \nu}\right)$. Then at least one of $\lim _{n \rightarrow \infty} \nu_{n}^{-}=$ $\nu$ or $\lim _{n \rightarrow \infty} \nu_{n}^{+}=\nu$ holds, and both hold if $r\left(S_{\mu \nu}\right)$ is irrational. Thus $S p e c_{\infty}[\mu]$ is a perfect set.

\section{Concluding Remarks}

Does there exist a value $\mu \neq 0$ for which $\operatorname{Spec}_{\infty}[\mu]$ is not a Cantor set? For algebraic $\mu \neq 0$ extra degeneracies for rational rotation intervals can occur, but we do not know whether there are ever enough of them to destroy the property of $\operatorname{Spec}_{\infty}[\mu]$ being totally disconnected. We numerically considered the test case $\mu_{0}=\frac{1}{2}$. Its full list of degenerate rotation intervals with denominator below 64 is given by $r=\frac{1}{5}, \frac{2}{7}, \frac{2}{15}, \frac{7}{20}$ and $\frac{11}{27}$, plus members of the sequence $r=\left\{\frac{2 n-1}{4 n}: n \geq 2\right\}$ given in Example 3.3 of part I. This list shows no sign of covering all rationals sufficiently near one particular point, as would be required if $\operatorname{Spec}_{\infty}[\mu]$ were not to be totally disconnected.

Another open question concerns whether the set of $\nu$ such that $T_{\mu \nu}$ has a nontrivial bounded orbit is of positive one-dimensional Lebesgue measure. The only case for which the answer is known is $\mu=0$, which gives measure 4 (the maximum possible for any $\mu$ ). This is a very special case, since for $\mu=0$, all rational rotation number intervals have length 0 . For $\mu \neq 0$, Theorem 4.2 shows that infinitely many of the rational rotation number intervals have positive length, so the uncovered measure is strictly smaller than 4. Numerical experiments suggest, in two different ways, that the measure is positive for at least some non-zero $\mu$. First, the calculation at $\mu=1$ of the uncovered area as a function of denominator suggested positive measure will remain. Second, numerical random selection of parameter values in the allowed interval seemed to produce invariant circles with positive probability.

\section{References}

[1] A. Avila and S. Jitomirskaya, The ten martini problem, eprint: arXiv:math.DS/0503363

[2] A. F. Beardon, S. R. Bullett, and P. J. Rippon, Periodic orbits of difference equations, Proc. Roy. Soc. Edinburgh 125A (1995), 657-674.

[3] J. Bellissard, Gap labelling theorems for Schrödinger operators, in: From Number Theory to Physics, (M. Waldschmidt, P. Moussa, J.-M. Luck, C. Itzykson, Eds.), Springer-Verlag: New York 1992, pp. 538-630. 
[4] J. Bellissard, Le papillon de Hofstadter (d'apres B. Helffer et J. Sjöstrand), Seminar Bourbaki, Vol. 1991/92, Astérisque No. 206 (1992), Exp. No. 745.pp. 7-39.

[5] P. Bougerol and J. Lacroix, Products of Random Matrices with Applications to Schrödinger Operators, Birkhäuser: Boston, 1985. (see p. 187ff)

[6] J. Fröhlich, T. Spencer, P. Wittwer, Localization for a class of one-dimensional Schrödinger operators, Comm. Math. Phys. 132 (1990), 5-25.

[7] M. Herman, Sur la conjugaison différentiable de difféomorphismes du cercle à des rotations, Publ. Math. IHES 49 (1979), 5-233.

[8] M. Herman, Sur les courbes invariantes par les difféomorphismes de l'anneau. Vol. 2, Astérisque No. 144, Soc. Math. France: Paris 1986, 248pp.

[9] D. Hofstadter, Energy levels and wave functions of Bloch electrons in a rational or irrational magnetic field, Phys. Rev. B 14 (1976), 2239-2249.

[10] S. Jitomirskaya, Metal-insulator transition for the almost Mathieu operator, Ann. Math. 150 (1999), 1159-1175.

[11] S. Kotani, Jacobi matrices with random potential taking finitely many values, Reviews in Math. Phys. 1 (1990), 129-133.

[12] J. C. Lagarias and E. Rains, Dynamics of a family of piecewise-linear area-preserving plane maps I. Rational rotation numbers, eprint: arxiv:math.DS/0301294

[13] J. C. Lagarias and E. Rains, Dynamics of a family of piecewise-linear area-preserving plane maps II. Invariant circles, eprint: arxiv:math.DS/0303007

[14] Z. Nitecki, Differentiable Dynamics: An Introduction to the Orbit Structure of Diffeomorphisms, MIT Press: Cambridge, MA 1971.

[15] L. Pastur and A. Figotin, Spectra of random and almost-periodic operators, Grund. Math. Wiss. 297, Springer-Verlag: Berlin 1992.

[16] J. Puig, Cantor spectrum for the almost Mathieu operator, Comm. Math. Phys. 244 (2004), 297-309.

[17] T. J. Rivlin, Chebyshev Polynomials: From approximation theory to algebra and number theory, Second Edition, John Wiley \& Sons, Inc., New York 1990.

[18] J. Sjóstrand, Microlocal analysis for the periodic magnetic Schrödinger equation and related questions, pp. 237-332 in: Microlocal analysis and applications (Motecatini Terme, 1989), Lecture Notes in Math. 1495, Springer-Verlag, Berlin 1991.

[19] B. Sutherland and M. Kohmoto, Resistance of a one-dimensional quasicrystal: Power-law growth, Phys. Rev. B 36 (1987), 5877-5886.

[20] A. Sütö, Schrödinger difference equation with deterministic ergodic potentials, in: Beyond Quasicrystals (Les Houches 1994), Springer-Verlag: New York 1995, pp. 481-549. 